\title{
That congestive cardiomyopathy is really hypertensive heart disease in disguise
}

\section{Chairman: J. DiCKINSON}

Dr Celia Oakley: I would like to propose the motion that congestive cardiomyopathy is hypertension in disguise. In congestive cardiomyopathy, we are looking for a cause of left ventricular failure. We can then explain the hypertrophy as a compensatory phenomenon following the dilatation of the left ventricle. The usual stimuli for hypertrophy, as we imperfectly understand them, will then exist.

On the other hand, we could be looking for a cause of left ventricular overload, in which case the failure could be secondary to the cause, whatever it is. The major stimulus for hypertrophy, apart from dilatation of the cavity of the left ventricle, is an increase in afterload. We know that the heart will accomplish volume work in a most efficient fashion with very little increase in metabolic demand and with very little hypertrophy, but pressure work is more costly. We all know that in aortic stenosis the heart hypertrophies and that in hypertension, it also hypertrophies. In congestive cardiomyopathy, we see a spectrum which varies from dilatation with minimal hypertrophy to much hypertrophy with less obvious dilatation but still there is always some dilatation of the cavity in these very big hearts which have been called 'idiopathic cardiomegaly', by the pathologists and which we describe as 'congestive cardiomyopathy'.

So let us consider the possibility that systemic hypertension provides the original cause for the hypertrophy with the secondary development of heart failure. We know that resistance is a function of pressure over flow and it is well known that when the flow goes down in heart failure the peripheral resistance goes up but the blood pressure usually stays about the same. In heart failure due to any cause with a low cardiac output the BP tends to be normal. We can now consider a situation where the BP was originally high; heart failure develops and the flow goes down but resistance stays the same and the high BP disappears. We are familiar with the fact that in heart failure due to any cause the peripheral resistance often exceeds the figure that exists in a patient who has high BP without heart failure.

Consider a left ventricle with a good thick wall, a high cardiac output and a good stroke volume ejected fast. The patient has hypertension, his heart is not dilated and on an X-ray it may look perfectly normal. We can consider two more situations, one is acute heart failure in response to the rather sudden development of high blood pressure, e.g. from renal ischaemia from an atheromatous plaque occluding the renal artery. Perhaps normotensive failure could supervene because the LV would be unable to sustain the sudden increase in its afterload. We can show this acute phenomenon haemodynamically, if we give methoxamine to a patient with a normal heart and suddenly raise the resistance to emptying of the left ventricle. Pulmonary oedema can result. The BP is high but falls again if failure is severe. If such a patient fails to recover and the resistance to flow remains high then his BP may never rise-he has congestive cardiomyopathy. If, on the other hand, he recovers from the load, he will recover with considerable hypertrophy, and, eventually he may develop high BP. We can imagine a spectrum of response to high BP which may either develop rapidly or may have developed slowly over a long period of time. We see patients who have hearts which are too big or ECGs which show inappropriately advanced LVH. It is common to see patients with rather large hearts which seem out of proportion to the severity of hypertension and the inevitable question arises: is something wrong with the left ventricle? Do they have congestive cardiomyopathy as well as hypertension? These, I suggest, are patients with hypertensive disease whose left ventricles have now become too defunct to produce hypertension. Their stroke volumes have diminished, their velocity of ejection has fallen and so the BP is lower despite no change in the peripheral resistance vessels.

We might get some support for this concept from epidemiology. Are hypertension and congestive cardiomyopathy frequent in the same areas? Professor Hutt told us that in his necropsy experience in Uganda hypertension was the commonest cause of cardiovascular death and he also pointed out the frequency of cardiomyopathy in that area. One can instance the same association in the West Indies, where hypertensive disease is frequent and often secondary to glomerulonephritis, and where there is also a high incidence of cardiomyopathy. We might ask ourselves, surely the pathologist can tell the difference from the histological changes in the arteries in hypertensive disease? But of course the 
changes in the arteries are a result of sustained hypertension and if the BP has been normal because the LV has failed to maintain output, then the changes in the vessels will not be present. Just as the clinician fails to find changes in the retinal vessels in such patients so the pathologist will fail to find support for the idea that the original disease was in the peripheral arteries rather than in the heart. Clinically, we also see patients with long-standing well-documented high BP who lose the need for hypotensive drugs at the same time as their hearts start to get larger and they develop failure.

In the patient who first presents with failure and normotension the diagnostic label will be congestive cardiomyopathy. It is not infrequent in COCM patients to have a history of hypertension having been found years before at an insurance examination. We see patents who on recovery from LVF develop high BP and we have several patients of this type who have recovered from a phase of failure with a large heart. With recovery, the heart shrinks and the BP goes up. Later they become able to support a high BP and they need hypotensive drugs. One such patient has been treated with massive doses of propranolol for about a year after being in severe trouble for months with a large left ventricle, normotension and a diagnosis of congestive cardiomyopathy. He now has a normal-sized heart but a very high BP, which was extremely difficult to control until he was treated with propranolol in large doses. He provides a very good extreme example of hypertensive heart failure masquerading as congestive cardiomyopathy and recovering to show his true colours. Now, if he had not recovered fully from his LV failure, which indeed is more common, he would still retain the label of congestive cardiomyopathy and would not have been diagnosed as hypertensive heart disease. So, sir, I submit that hypertensive heart disease is disguised in the condition which we call congestive cardiomyopathy and that in COCM the disease is really in the peripheral arterial tree and not primarily within the myocardium.

DR DICKInson: Thank you very much. Dr Oakley is going to be supported further by Dr Ian Brockington.

DR I. Brockington: I would like to say something about two forms of myocardial failure which occur in coloured people with a mildly raised blood pressure which falls on treatment to normal levels. These are post-partum heart failure and Nigerian 'heart muscle disease'. Post-partum heart failure was first described in 1938 by Hull \& Hidden. It occurs in coloured women, usually multiparous, who present about 6-8 weeks after a normal pregnancy, usually make a good response to treatment and leave hospital with a normal heart and a normal blood pressure. They tend to relapse after further pregnancies and they occasionally develop chronic hypertension. Nigerian 'heart muscle disease' is a much more common disorder, which occurs in middle-aged Nigerian men and women and accounts for about $15 \%$ of medical admissions in this age group. There is an insidious onset of the symptoms of biventricular failure. $70 \%$ have a raised blood pressure which usually falls on treatment, and the prognosis is good, or at least better than any other heart disease except frank hypertensive failure. One might add, in a provocative spirit, that South African 'cryptogenic heart disease' is similar in all respects to Nigerian 'heart muscle disease'.

These diseases present with a mild elevation of blood pressure which falls to normal with successful treatment of heart failure, and we do not know anything about the patients up to the moment of presentation. There are two possibilities. One theory holds that the pathology is in the myocardium, and that the elevation of blood pressure is secondary to heart failure and, of course, only lasts as long as heart failure itself. This has been called, at Ibadan, 'reactive hypertension', but more generally it has, I think, been termed 'Sahli's hochdruckstauung' (Sahli, 1901). The other theory takes a leap into the dark and proposes that these patients have beeng severely hypertensive in the past and that hypertension is the sole cause of heart failure in these patients. The first hypothesis has held the field so far. This may be because these diseases have been classified as 'cardiomyopathies'. This is a word which has been used by pathologists as a rubric for intrinsic myocardial diseases of various kinds. When it is used by clinicians, there is a tendency for a prejudice to be established in favour of myocardial disease as opposed to cardiac overload which would equally well explain the clinical picture. There is no doubt that in heart failure of any cause the peripheral resistance rises in order to maintain the blood pressure when the cardiac output falls. The issue is whether the mean pressure itself rises. Now there are several agents acting in heart failure which might tend to produce a raised mean pressure in a normal person, e.g. sodium retention, carbon dioxide retention, anoxia, reduced renal blood flow, anxiety and catecholamine release, but there is no published evidence to show that this occurs in patients with heart failure. Nor can I think of any reason why this should happen only in idiopathic heart diseases. If hypertension complicated heart failure or left ventricular failure then it should be found generally in heart failure and this is not so. In Ibadan, sixty miscellaneous cases of heart failure presented with a pressure of $120 /$ $80 \mathrm{mmHg}$. However, I would take the argument further and claim that this mechanism is impossible. How can a left ventricle which goes into failure under a normal load, which is dilated, feebly contracting, 
with mitral incompetence and pulmonary hypertension and secondary right heart failure-how can that ventricle sustain an increase in its load, imposed by a disproportionate increase in the peripheral resistance? Surely that would cause either catastrophic pulmonary oedema or such a severe increase in heart failure that there would be a further fall in cardiac output pari passu with the increase in systemic resistance, so that the ventricle would not in fact achieve a higher mean blood pressure. I have written down these arguments in the American Journal of Cardiology (Brockington, 1971) and am still waiting for the refutation.

Now let us consider the other possibility, that the patients have been hypertensive in the past. This seems to me haemodynamically sound. The main difficulty is explaining why the blood pressure falls in hypertensive failure and I can only speculate about the reasons for that. But I am quite sure that it does occur. Figure 1 shows a Nigerian patient who presented in heart failure in 1957 with a blood pressure of $180 / 130 \mathrm{mmHg}$. He was treated with various drugs and improved, and then defaulted and came back twice in heart failure with a blood pressure of $140 / 100 \mathrm{mmHg}$. When treated with digoxin and diuretics and bed rest, his blood pressure remained low (which we find is usually the case) and he then unwisely defaulted again, and the third time he came back with a blood pressure of $110 / 90 \mathrm{mmHg}$ in severe failure. If he had come for the first time at this stage, he would have been a classic case of normotensive 'heart muscle disease' ('idiopathic cardiomegaly', 'primary myocardial disease', 'congestive cardiomyopathy' or what you like). He died, and his kidneys showed moderate afferent arteriosclerosis, confirming that he had chronic hypertension. This individual case demonstrates that the blood pressure can fall in the course of severe hypertensive failure.

I was very interested, listening to John Fowler yesterday, to hear that in Uganda, scarcely any patient who presents with hypertensive failure dies of heart disease. That has also been our experience at Ibadan (Brockington \& Edington, 1972). It really is remarkable that a disease which drives the patient into a state of biventricular failure should have a negligible death rate (from heart disease) and I would suggest that the explanation is that frank hypertensive failure is mild hypertensive failure. This is a hypothesis which can be tested by a study of arteries and at Ibadan we have made a start with that. We have looked at the kidneys and the retinal arteries and the size of the aorta (Brockington \& Bohrer, $1970)$ and we have also looked at the voltage on the electrocardiogram in these patients with chronic 'heart muscle disease'. I have no time to present that evidence now, but it confirms this hypothesis in part. There are cases of normotensive heart muscle disease with clear-cut evidence of hypertension. Equally, there are patients who do not have evidence

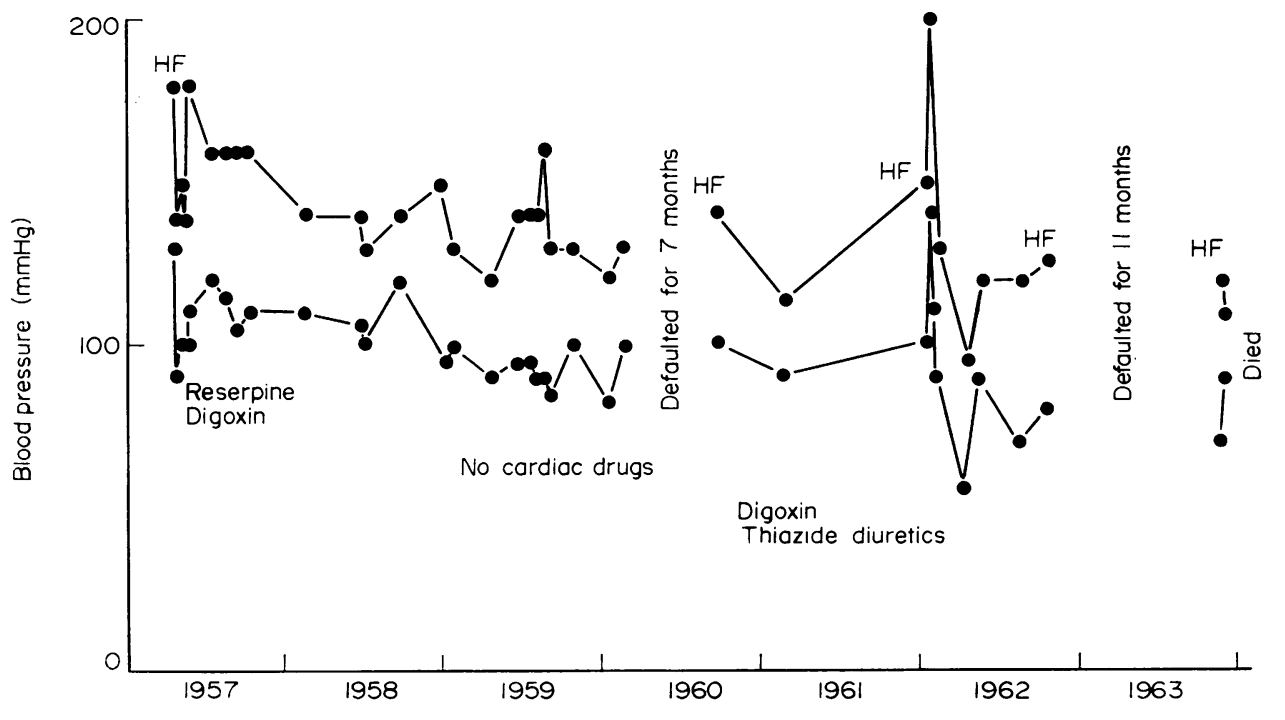

FIG. 1. The course of the blood pressure in a patient who presented with hypertensive heart failure (BP 180/130) and, after defaulting twice, eventually died with a picture of normotensive 'heart muscle disease'. HF, heart failure. 
of hypertension and therefore we can be fairly sure that they are not cases of chronic hypertensive heart disease.

That brings me back to the problem of postpartum heart failure. This is a disease which occurs after a normal pregnancy, usually without signs of toxaemia. We can be sure that the patients have not been hypertensive in the past. Heart failure is acute, so, if it has a hypertensive basis, that basis is acute hypertension, and there must be (if this theory is correct) a form of hypertension which corresponds exactly with that of the heart failure. It must be common in Negro women and almost unknown in whites. It must reach a peak about 6 weeks after a normal pregnancy. It must be self-limiting as a rule but tend to recur after subsequent pregnancies and occasionally lead to chronic hypertension. These are precisely the characteristics of post-partum hypertension as described by Stout in 1934 and confirmed by several other American obstetricians. In Nigeria, where I studied 100 consecutive patients in Professor Lawson's clinic, I found that $18 / 100$ developed postpartum hypertension after a normal pregnancy.

A trial of prophylactic therapy would test this theory, and I would predict that, if obstetricians will follow carefully the blood pressure of certain susceptible groups (and I mean coloured women who have had at least six children or a previous episode of post-partum heart failure) and will treat them prophylactically for hypertension, they will very much reduce the prevalence of this heart disease. And I think that if this hypothesis is confirmed, it will have wider implications because it will offer a paradigm for the clinical and pathological picture of acute hypertensive heart failure, which has so far been lacking.

\section{Acknowledgments}

I would like to thank the Wellcome Trust, who financed this work, and all those at University College Hospital, Ibadan, who made it possible. I also thank Raymond Carlisle for translating the article by Sahli (1901).

\section{References}

Brockington, I.F. (1971) Postpartum hypertensive heart failure. American Journal of Cardiology, 27, 650.

Brockington, I.F., and Bohrer S.P. (1970) Enlargement of the aortic shadow in Nigerian heart muscle disease. Acta cardiologica, 25, 344.

Brockington, I.F. \& Edington, G.M. (1972) Adult heart disease in Western Nigeria; a clinicopathological synopsis. American Heart Journal, 83, 27.

Hull, E. \& Hidden, E. (1938) Post-partum heart failure. Southern Medical Journal, 31, 265.

SaHLI. (1901) Herzmittel und Vasomotorenmittel. Verhandlungen des Congresses fur innere Medicine, 19, 45.

Stout, M.L. (1934) Hypertension 6 weeks post-partum in apparently normal patients. American Journal of Obstetrics and Gynecology, 27, 730.
DR Dickinson: Thank you very much Dr Brockington. Before we start our general debate, I will ask Professor Goodwin to put the other side of the argument.

Professor J. Goodwin: The motion we are debating is whether heart failure and hypertrophy develop as a result of primary myocardial disease or whether the hypertrophy comes first as a result of hypertension followed by the heart failure. There are two major groups: hypertrophy secondary to the afterload (pressure load) which has been imposed by hypertension, and primary myocardial disease, in this instance congestive cardiomyopathy.

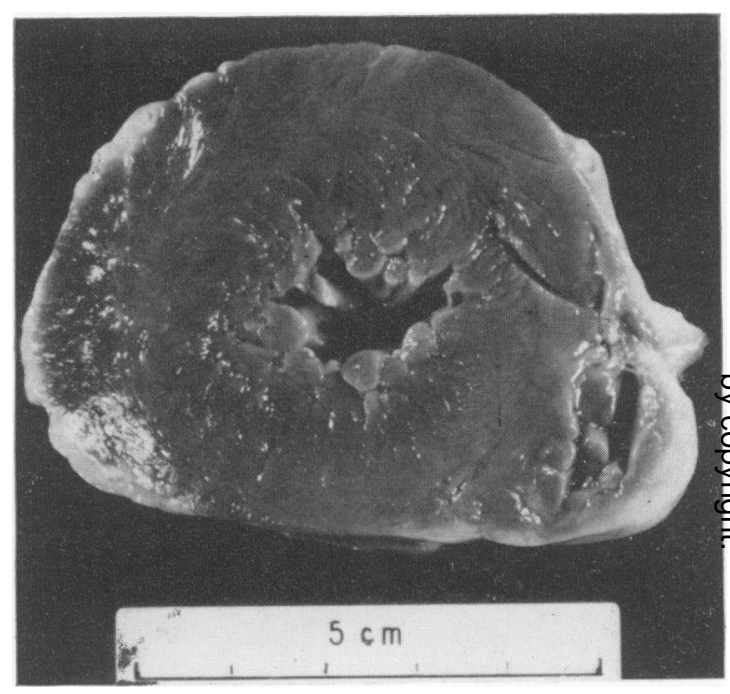

FIG. 1. Section of heart in hypertensive heart disease, showing massive hypertrophy of the left ventricle and small cavity (courtesy of Dr E. Olsen).

In Fig. 1 you will see a hypertensive heart with massive hypertrophy and a moderate-sized cavity. This is the hallmark of hypertensive heart disease. The heart progressively hypertrophies against the pressure load and then eventually, if the pressure load is not relieved, it will fail. But it does not fail until very considerable hypertrophy has developed, unless there is some other disease as well. Figure 2 shows the heart of congestive cardiomyopathy with its greatly dilated ventricle and its inappropriate degree of hypertrophy. These are not of course comparable, for they do not show the hearts presented in the same way. Nevertheless, I think that we would all agree that in congestive cardiomyopathy the hypertrophy is relatively less than the degree of cavity dilatation, in contrast to hypertension, which is the very striking difference to my mind between the two conditions. The concept of eccentric and 


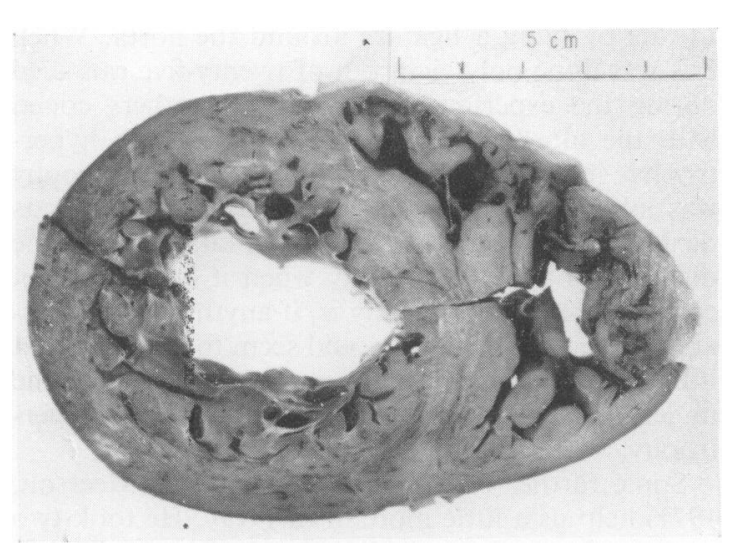

FIG. 2. Section of heart in congestive cardiomyopathy, showing dilated cavity of the left ventricle and secondary hypertrophy (courtesy of Dr E. Olsen).

concentric hypertrophy has been revived by Grant and his colleagues from Buffalo (Grant, Greene \& Bunnell, 1965). Pure concentric hypertrophy denotes an increase in muscle mass without increase in cavity size; this is the type of thing we see for example in hypertension or aortic stenosis. Eccentric hypertrophy occurs when there is dilatation secondary to hypertrophy. Figure 3 shows the ratio of thickness of the wall of the ventricle to the mean radius of the cavity. Those patients with an afterload such as valvar obstruction or hypertension have a very greatly increased ratio as compared to those with myocardial disease. In his experimental work on

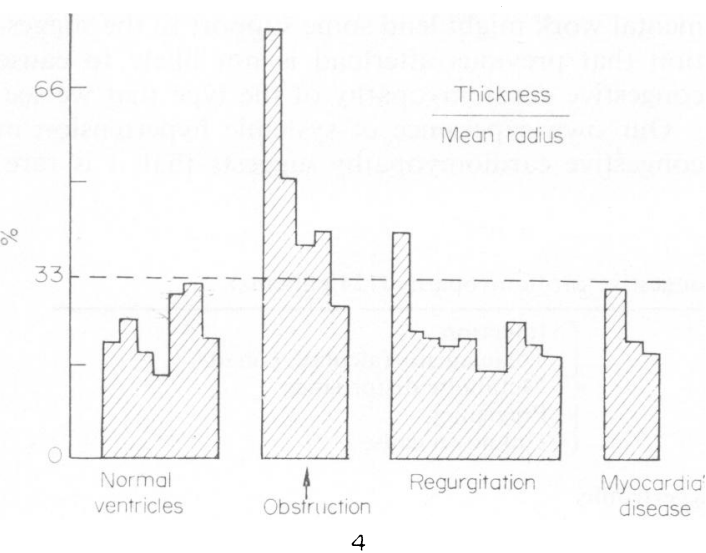

Fig. 3. Ratio of left ventricular wall thickness to cavity size determined by angiocardiography in normal hearts, left ventricular outflow obstruction, valvar regurgitation, and myocardial disease. Note the greatly increased ratio in ventricles with obstruction, which (after load) compared with those with myocardial disease in hypertrophy, is less striking, owing to cavity dilatation (from Grant $e t$ al. (1965) by permission of the author and editor). cardiac hypertrophy, Meerson (1969) has described three phases of the process; damage caused by the afterload (usually produced in the animal by tying a ligature around the aorta or pulmonary artery), is followed by rapid hypertrophy of the ventricle. But initially the phase of hyperfunction is followed by the phase of hypertrophy which may remain stable for some time but eventually leads on to a stage of exhaustion or failure. This pattern corresponds to the normal progression of hypertensive heart disease, if the hypertension is not treated. In congestive cardiomyopathy, we do not recognize and have never seen any stage of hyperfunction or hypertrophy but usually one of progressive congestive heart failure over a relatively short period. However, in some patients we cannot exclude a stable period but without the initial phase of hyperfunction and without marked hypertrophy. The angiographic characteristics of congestive cardiomyopathy have been already shown to you by Dr Oakley, but I would remind you of this very grossly enlarged cavity size with a relatively modest degree of hypertrophy which is quite different from the massive hypertrophy with little, if any, dilatation of the hypertensive heart. On cine-angiography in congestive cardiomyopathy we can see that the contraction of this damaged ventricle is very poor, the ejection fraction is reduced and the diastolic volume increased. The thickness of the free wall of the left ventricle is not significantly increased despite the fact that the cavity is very much dilated. Our angiographic studies (Croxson \& Raphael, 1969) have shown that patients who have died after a short history have a relatively slight increase in left ventricle wall thickness, as compared with patients who lived longer. And estimates of the relation of wall-thickness to cavity-size confirm this, showing a disproportion between the large cavity and the thickness of the wall. Again, this is quite unlike the pattern of hypertensive heart disease. Studies of ventricular volumes by angiographic means (Croxson \& Raphael, 1969) have shown that when the ratio of cavity-size to wall-thickness is high, patients die sooner than when hypertrophy causes a lower ratio of cavity to wall (Fig. 4); again suggesting that dilatation due to myocardial damage is the cause of hypertrophy and failure, rather than the reverse, as in hypertensive heart failure. The left ventricle in congestive cardiomyopathy functions at a high enddiastolic volume and an elevated end-diastolic pressure with an early diastolic filling gallop. This gallop is not found in hypertensive heart disease until the late stages when considerable hypertrophy has already developed and has been followed by dilatation-the reverse of the situation seen in congestive cardiomyopathy. This scheme (Table 1) purports to set out the various processes in conges- 


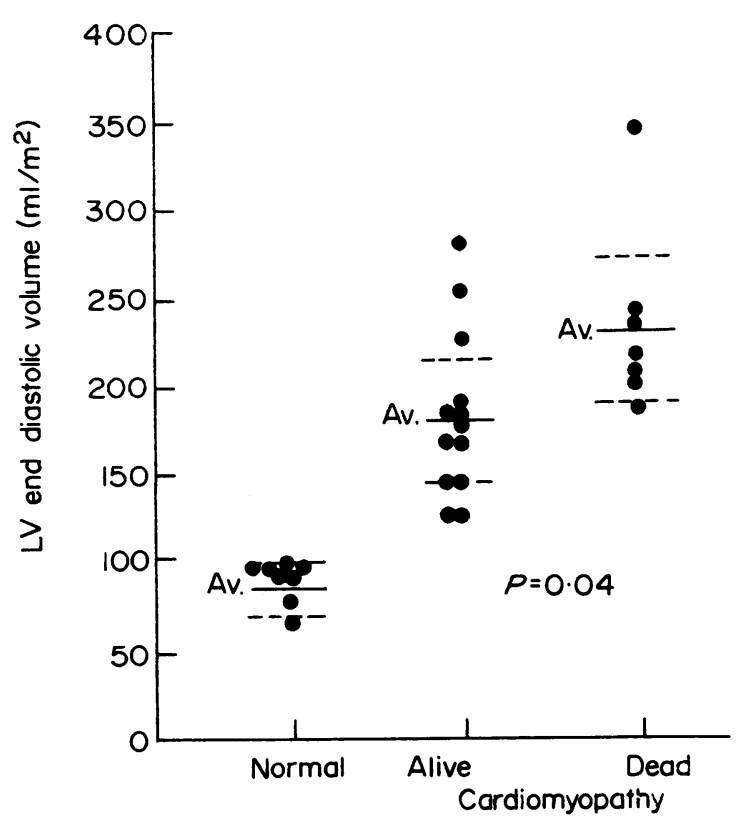

FIG. 4. Ratio of wall-thickness to cavity-size in congestive cardiomyopathy measured by angiocardiography, in a personal series of patients. Note that those patients with the greater wall-thickness survived longer than others with a smaller ratio (courtesy of Dr M. Raphael and Dr R. Croxson).

tive cardiomyopathy and I wish merely to emphasize that the evidence suggests that the initial insult damages the ventricle, which then dilates; that there is a relatively poor response to hypertrophy and that death follows from relentless congestive failure (Goodwin, 1970). It is interesting in this context to draw attention to some Russian experimental work in which hypertrophy of the left ventricle was induced in rats by tying a ligature around the aorta. When this was done only eight out of twenty-five rats died during the experimental period; the others coped with the afterload very well by developing hypertrophy (Meerson, 1969). But when hypertrophy was inhibited by actinomycin D, nearly all the rats died. Perhaps I might suggest tentatively that the development of hypertrophy when it does occur in congestive cardiomyopathy is, if anything, a favourable feature, and thus it would seem to be likely that if hypertensive heart disease in disguise had been the underlying cause there would have been gross hypertrophy.

Some further experiments of Meerson (Meerson, 1971) help us a little more in this way. He took two groups of rats, those untrained and those trained to hypoxia on effort (Fig. 5). The hatched columns show rats in whom a ligature has been tied round the aorta so that they had an afterload imposed on the left ventricle. The vertical axis represents the contractile function of the left ventricle and you will see that in untrained rats the power of the left ventricle was much more reduced than in those who had had the coarctation induced, but in those who had been trained afterwards the degree of reduction in contractile force was very much less.

Now you may ask what relation this has to con gestive cardiomyopathy and hypertension and suppose the answer is, very little, except that George Burch has claimed that prolonged bed-rest has some benefit in congestive cardiomyopathy and we know from our studies that persistent physical activity tends to exacerbate congestive heart failure in congestive cardiomyopathy. So, perhaps this experimental work might lend some support to the suggestion that previous afterload is not likely to cause congestive cardiomyopathy of the type that we see.

Our own experience of systemic hypertension in congestive cardiomyopathy suggests that it is rare,

TABLE 1. Schema to indicate the pattern of congestive cardiomyopathy (124 patients)

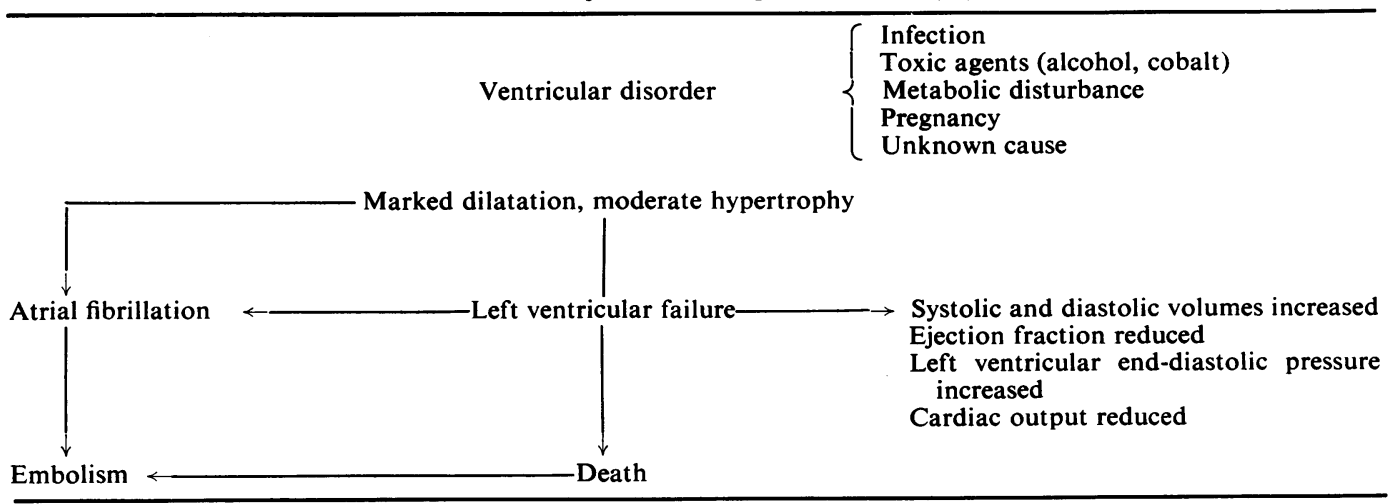

Infection

oxic agents (alc

Pregnancy

Unknown cause

Systolic and diastolic volumes increased

Left ventricular end-diastolic pressure increased

Cardiac output reduced 


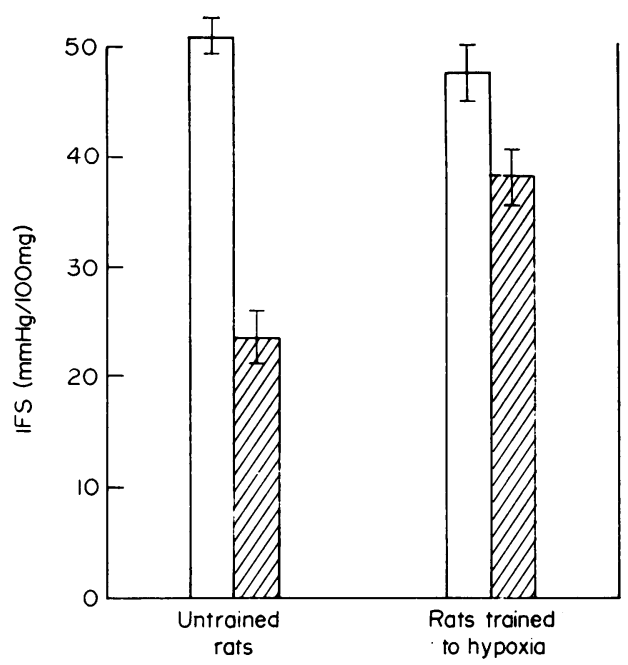

Fig. 5. To show the effect of training to hypoxia on the disease in maximal force and contraction (IFS) of the myocardium produced by aortic coarctation in rats. In untrained rats the maximal contraction force is decreased by more than half after coarctation, but the decrease after training is not significant. (From Meerson, F.Z. (1971) British Heart Journal, Suppl. 33, 100. By permission of the author and publishers.)

only nineteen of seventy-four patients studied recently by Dr Arni Kristinson (Kristinson, 1969) from our unit had a blood pressure of greater than
$149 / 90 \mathrm{mmHg}$ in the past or during the period of study, and I would suggest that it is rather unlikely that systemic hypertension is the cause of congestive cardiomyopathy when the majority of patients with congestive cardiomyopathy have never been hypertensive. Those who were hypertensive could be divided into four groups. (1) Those whose blood pressure later became normal after treatment and perhaps in those catecholamine excess or poor renal blood flow might be the cause during the heart failure. (2) There were some patients whose blood pressure was initially normal and later became elevated. (3) There were patients who were constantly hypertensive, again possibly as a result of heart failure; and (4) those who were constantly hypertensive and who perhaps bear out Dr Oakley's view that in some patients there may be a background of hypertension in congestive cardiomyopathy; I would not seek to deny this for a moment. As regards the average total duration of the illness it was significantly greater in the hypertensive patients than in those who were normotensive, and the hypertensive patients who were studied angiographically did appear to have a greater degree of wall thickness so this hypertrophy perhaps helped them, but again, this would seem to be a little unlikely if they were really suffering from hypertensive heart disease, a condition known characteristically to cause hypertrophy of the left ventricle because of the afterload imposed by the hypertension. If we look a little further into twelve of these patients we do not really

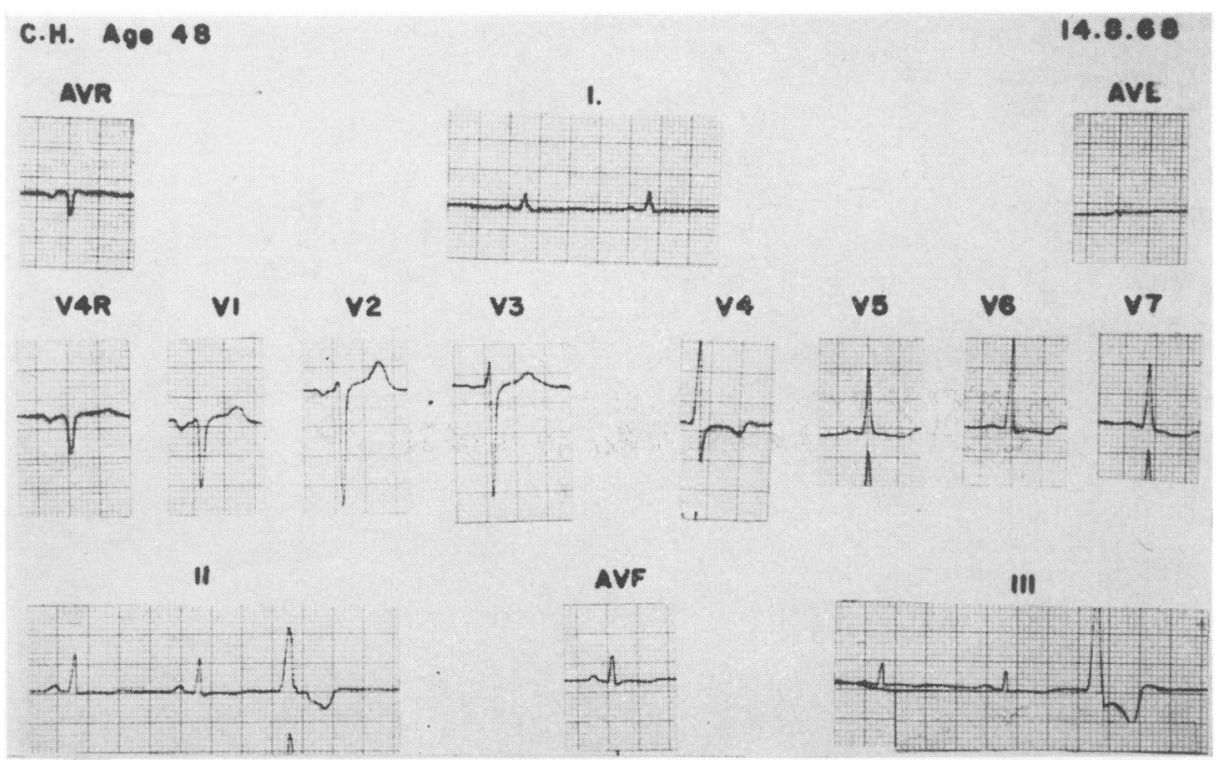

FIG. 6. Electrocardiogram (male, aged 48 years) in constrictive cardiomyopathy, showing low voltage QRS complexes and flat $\mathrm{T}$ waves (repolarization changes only). Ventricular ectopic beats are present in leads II and III. 
see evidence of previous hypertension or hypertensive disease. There was no family history, and no past history of renal disease. Most of them had a third heart sound, again unusual in hypertensive heart failure except in the later stages. There was no urinary abnormality and retinopathy was present in only two and was mild in degree. Necropsy in patients who die of congestive cardiomyopathy does not show changes in the kidneys suggestive of longstanding hypertensive disease. So that in the majority it does not seem as if longstanding hypertension has been present or has damaged the vascular system. Further evidence may be obtained from the ECG which in congestive cardiomyopathy usually shows rather low voltage, flat and slightly inverted $T$ waves and a non-specific pattern of myocardial damage, often with intra-ventricular conduction defect(Fig.6). The ECG of systemic hypertension, however, by the time the patient has gone into heart failure, shows marked left ventricle hypertrophy of the systolic overload type with repolarization changes and high voltage of the QRS complexes in left praecordial leads (Fig. 7). So that there is also a striking difference electrocardiographically between the two groups. There is a small group of patients that $\mathrm{Dr}$ Oakley describes who appear to lose their hypertension spontaneously and have large hearts, and who she thinks have hypertensive heart disease and I think have congestive cardiomyopathy. But there are really only two main reasons why patients lose their hypertension over a period. One is therapy, $\stackrel{\hookrightarrow}{\Rightarrow}$ which we are not concerned with in this context, and $\underset{\sim}{\vec{F}}$ the other is major cardiac infarction, which it is well known will reduce the blood pressure to normal, 흠 sometimes for many months. Patients with congestive $\frac{\bar{m}}{\bar{c}}$ cardiomyopathy do not have evidence of myocardial $\mathbb{\nabla}$ infarction or coronary thrombosis as may patients with hypertensive heart disease. The coronary os arteries in cardiomyopathy are smooth and regular $\vec{\circ}$ in all the patients that we have studied and also in $\overrightarrow{.}$ those that Dr Eckhardt Olsen has studied. And so $\vec{\omega}$ here we have yet another difference; whereas hyper- -0 tensive heart disease is often associated with coronary artery disease, congestive cardiomyopathy seems only very rarely to be associated with coronary artery $\infty$ disease. Now I would submit, although in some patients with congestive cardiomyopathy hypertension is present, it is a factor the significance of $\forall$ which we do not know. I would further submit that o the suggestion that congestive cardiomyopathy is merely hypertensive heart disease in disguise flies in $\overrightarrow{0}$ the face of all the evidence, scanty though that $\stackrel{\mathbb{D}}{\circ}$ evidence may be. And I am sure that such an $\frac{\Phi}{3}$ intelligent and sophisticated audience as yourselve will have no hesitation in rejecting utterly the thesis of my learned friends.

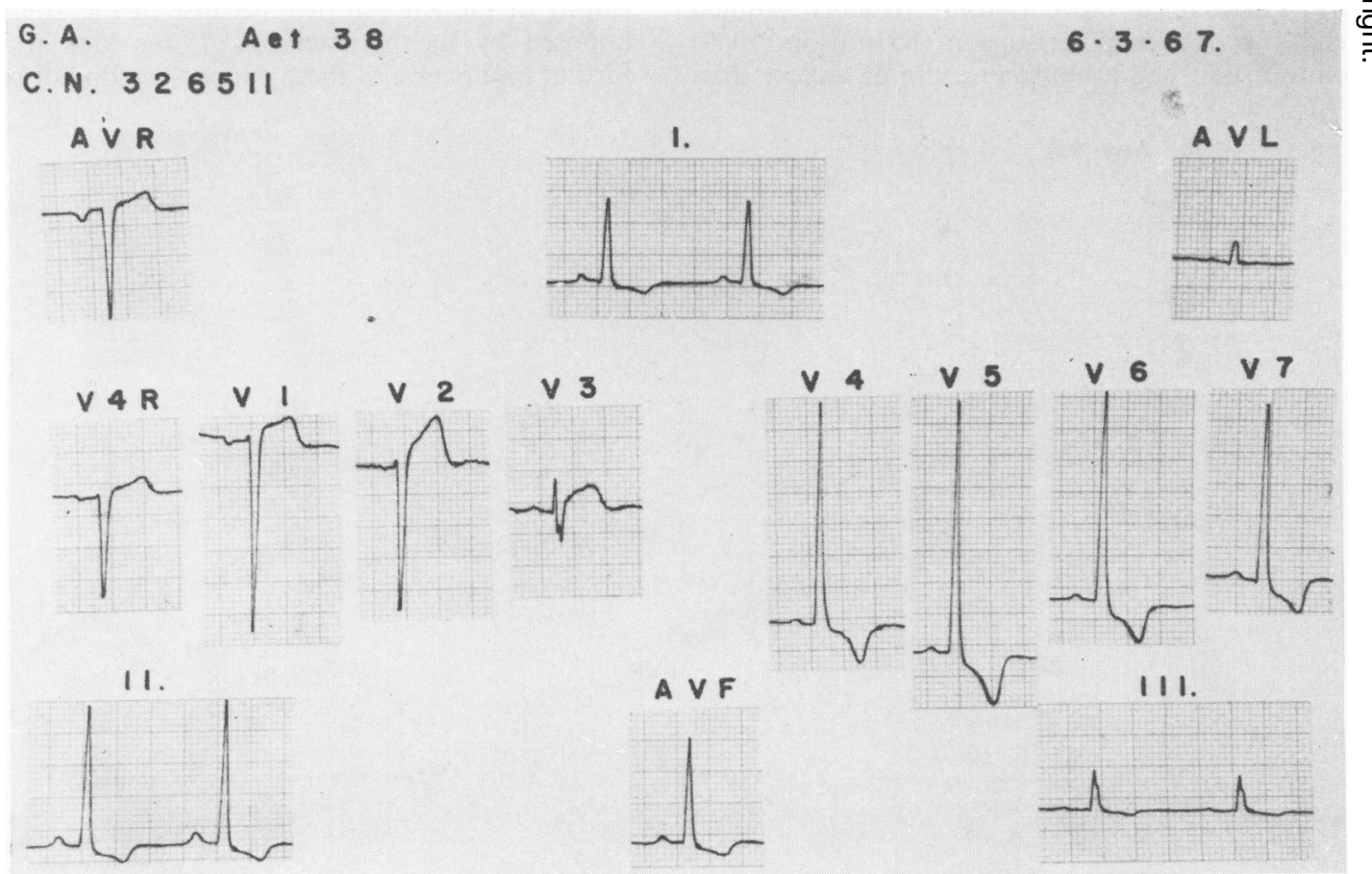

FIG. 7. Electrocardiogram in hypertensive heart disease showing high voltage of QRS complexes and deep T wave inversion indicating systolic (pressure) overload of the left ventricle and severe left ventricular hypertrophy. 


\section{References}

Croxson, R.S. \& Raphael, M.J. (1969) Angiographic assessment of congestive cardiomyopathy. British Heart Journal, 31, 390.

Goodwin, J.F. (1970) Congestive and hypertrophic cardiomyopathies: A decade of study. Lancet, i, 731.

Grant, C., Greene, D.G. \& Bunnell, I.L. (1965) Left ventricular enlargement and hypertrophy. American Journal of Medicine, 39, 895.

Kristinsson, A (1969) Diagnosis, natural history and treat- ment of congestive cardiomyopathy. PhD. Thesis, University of London.

MeERSON, F.Z. (1969a) The myocardium in hypertension, hypertrophy and heart failure. Circulation Research, 25, Suppl. II, 6.

MeERSON, F.Z. (1969b) (quoting Rozanora, L.S.) The myocardium in hyperfunction, hypertrophy and in heart failure. Circulation Research, 25, Suppl. II, 35.

MeERson, F.Z. (1971) Mechanism of hypertrophy of the heart and experimental prevention of auto cardiac insufficiency. British Heart Journal, 33, Suppl. 100.

Another possibility is that we are looking at the overlap of distribution curves of blood pressure in the general population and the distribution of myocardial function. If a patient has both a rise in pressure and poor myocardial function for some reason, he is very much more likely to fail than somebody with the same blood pressure who has good myocardial function. I know that I am using these words in a very imprecise manner but $I$ am not sure that I can make them more precise. In some cases, poor myocardial function might be due to an obvious cause, such as ischaemic heart disease or other known causes of heart muscle disorder. If there is a wide distribution curve for heart muscle function in the apparently healthy population, and we know that there is a wide distribution curve of pressure, then you might get some of these apparently puzzling cases when the two occurred together by chance.

There are other possible explanations besides those of primary myocardial disease, which I agree with Professor Goodwin is the most likely. It is possible that we could be looking at some kind of disorder of circulatory control in congestive cardiomyopathy and I would instance two observations which I think are interesting. The first is an observation recently made in Cleveland, Ohio, concerning the distribution of labelled angiotensin in heart muscle. 1-3 min after injection, you find the angiotensin bound to the nuclear membrane of myocardial cells. These workers went further and showed that angiotensin given in this manner caused stimulation of nucleoprotein synthesis in the myocardium. Now I don't think these experiments have been taken to the point where one can dissociate this from the effect of the afterload on the ventricle caused by raising pressure but there may be a direct effect of angiotensin upon the muscle cell in stimulating protein synthesis. I suppose that one could begin to think of a situation whereby a high level of angiotensin might stimulate myocardial hypertrophy directly and on some occasions disproportionately, rather than by its effect on blood pressure.

The other possibility is a very much longer shot. I was talking just a few days ago to some people who are studying muscular dystrophies in children and lend much support. 
they now hold the view that muscular dystrophy is not primarily a disorder of muscle but is a disorder of the nerve supply of muscles. Besides the motor nerve to muscles releasing acetylcholine at the motor endplate they also release a trophic protein and the evidence for the existence of this is derived partly from cross-innervation studies. You can now crossinnervate a slow and a fast muscle. The slow one becomes a fast muscle, the fast one becomes a slow; in other words, the nerve supply controls the properties of the muscle to a considerable degree. If you could envisage that the sympathetic nerve supply to the heart, perhaps not only the sympathetic nerves, might also secrete a trophic protein, you could envisage that this might be a mechanism for disproportionate hypertrophy of the heart muscle. I was impressed by some demonstrations by Professor Pearse who showed disorderly overgrowth of the sympathetic nerve endings in the muscle of patients with cardiomyopathy. If they were releasing excessive amounts of a stimulating protein or polypeptide then you might begin to have an explanation. As you can see, Dr Dickinson, I have skilfully avoided coming down on one side or the other and I am content to continue to avoid doing this.

DR Dickinson: Right, thank you very much Professor Dollery. I think all of us agree that we should certainly not come down solidly on one side or the other. My feeling is at the moment that if I were in Nigeria I would support Dr Oakley almost $100 \%$, and if I were at Hammersmith Hospital I would support her about $30 \%$ !

I would like to just take up one point made by Professor Dollery, his two interesting speculations at the end. There is a difference, at least in rabbits in my experience, between the effects on the heart of long-continued infusions of noradrenaline compared with long-continued infusions of angiotensin. Angiotensin infusions long-continued produce hypertrophy of the muscle, but when you are producing similar levels of BP maintained for a long time by infusions of noradrenaline, you don't get hypertrophy-you tend to get diffuse myocardial damage, and a dilated flabby heart, almost perhaps congestive cardiomyopathy, except that the rabbits did not really go into left ventricular failure. Dr Oakley drew attention to the fact that sometimes patients who have congestive cardiomyopathy for a long time turn into frank hypertensives as their heart seems to strengthen up. I think that one should bear in mind that this may not represent the evolution of the disease but possibly a new factor coming in, and that is renal infarcts. As you know, there is an increased incidence of hypertension in mitral stenosis, which seems rather unusual. I think this is usually attributed to renal infarcts. Patients who have been in bed for long periods with congestive cardiomyopathy could well form some renal infarcts and subsequently raise their blood pressure. I personally have seen all the sequences of events that have been mentioned by Dr Oakley, Dr Brockington and Professor Goodwin and I believe all of them! It is just a matter of assigning frequency and statistical probabilities in different communities.

DR Hollman: I would like to attack both sides in this debate because the more I think about the classification of cardiomyopathies the more confused I become. Professor Goodwin and Dr Oakley have only got themselves to blame for getting into this argument, because they have erected an edifice of classification which really has no substantial basis. To use a botanical analogy, it is as if they tried to classify plants according to their colour, and you know how hopeless and un-scientific that is. There is one important fact about the whole subject we have been discussing and that is that heart muscle disease can occur with no obvious aetiology. Some of these patients have thick hearts and some have thin hearts, and perhaps it should be left at that. To build up an edifice of congestive cardiomyopathy, hypertrophic obstructive cardiomyopathy, hypertrophic non-obstructive cardiomyopathy and, much worse to my mind, constrictive cardiomyopathy (which I think is a terrible designation) leaves me completely flabbergasted. I think that this is a totally artificial argument and that the real problem is lack of knowledge of the aetiology. It must be only too clear to Dr Oakley that at least some of her cases have an infective basis and she has not touched on that at all. I am sorry to have attacked both the protagonists but I think they are talking about something that doesn't exist!

DR SHAPER: There is a tendency for many people who work in Africa to focus on the problem of many diseases occurring in the same patient. There is also the problem of putting a whole series of quite disconnected things together in order to make an edifice. Let us look at the post-partum problems in this context. There is a problem of African women or Negro women in the southern United States who go into heart failure having been perfectly normal before their pregnancy and during their pregnancy. They do not always have hypertension, so there are two separate problems: there is hypertension and there is heart failure both developing in African women after delivery, and they do not always run together. A recent publication from Kampala describes African women who had high blood pressures at the time of delivery and who were followed afterwards. Three months after delivery, a large group of them were still hypertensive. They all had renal biopsies and the vast majority had underlying glomerulonephritic lesions. So in that group, there was a renal cause for the high blood pressure. 
We were not dealing with some generalized blood pressure-heart hypertrophy phenomenon.

Again, in African hospitals one sees many patients admitted with high blood pressure and renal disease, who die in uraemia with or without heart failure. This is a very selected group, because when one goes out into the community and does field surveys, one finds very few of these cases. They are the ones who tend to come into hospitals and die, usually before they are 40 . Blood pressure patterns in the community in Uganda and Nigeria are very similar to the blood pressure patterns in this country. So again, you have populations with a lot of high blood pressure as you have here. I agree with the remark made by Professor Dollery; the frequency of raised blood pressures in the patients with congestive cardiomyopathies probably reflects the fact that many people in this country and in the tropics have elevated blood pressures.

DR BRockington: I think Dr Shaper is right on one point. Each of these diseases has to be considered separately. Nigerian 'heart muscle disease' and post-partum heart failure are quite distinct problems. One is predominantly a chronic disease whose basis would have to be (if hypertensive) chronic hypertension, and the other is an acute disease. But the haemodynamic arguments against the possibility that hypertension is a complication of heart failure apply to them both.

It's true that some patients with post-partum heart failure present with a normal blood pressure. Eleven out of fifty Nigerian cases did so. I don't mind if you exclude those. They are only a small number, though personally I would prefer to take the entire group of patients with biventricular failure presenting in the post-partum period after a normal pregnancy-I think that is a sounder policy. If severe hypertension causes failure and the blood pressure then falls to a mildly hypertensive level, I don't see why it shouldn't fall all the way to a normal level. There is some evidence from the outcome of these patients (though it's a very small series) that the lower the blood pressure the more severe the failure. Patients who take longer to recover from heart failure have had lower presenting blood pressures. And this, if you like, adds some weight to the view that normotensive patients are the severe end of a spectrum.

I am on thin ice when talking about South African 'cryptogenic heart disease', because I have never seen a case. But, if it's true that it's associated with mild transient hypertension, that is an important fact, which shouldn't be forgotten, and can't be shrugged off as secondary to heart failure. If the prevalence of hypertension in these patients is greater than in the general population (as it is in Nigerian 'heart muscle disease' with a $70 \%$ preva- lence of hypertension compared with $15 \%$ in people of the same age group), that's got to be explained. Now I believe that hypertension is very common in the South African Bantu, and this poses a problem. The presence of hypertension in "cryptogenic heart disease' may be a coincidence. Equally, widespread hypertension would account very well for a very prevalent heart disease. So the detailed figures for hypertension of different levels in different age groups and different ethnic groups are of great interest here.

DR VAN DE WALL (Groningen): I agree with Dr Hollman when he says that we may be creating artificial terms for diseases of unknown aetiology. In a recent study of thirty patients with HOCM I have never seen the transition of HOCM to COCM. Clinically there is a difference; it is not an artificial division which we are making to create problems for ourselves and the patient. We are using concepts of hypertrophy in a clinical context whereas the concept of hypertrophy is based on anatomical and pathological concepts. The criteria for hypertrophy are based upon wall thickness and on histological criteria. In the case of HOCM the hypertrophy is needless; there is no increased afterload. The secondary form of hypertrophy we can cover with the concept of homeometric autoregulation; it is a functional haemodynamic concept and not a pathologic concept. How can we be sure that on chest radiography in eccentric hypertrophy, that the heart muscle itself is hypertrophic in a clinical or pathological sense? The most confusing aspect of the HOCM for me personally is in considering the reasons we have to use the adjective 'hypertrophy'. It is the reverse of the eccentric hypertrophy for it is a thick wall ventricle; it has a small volume and functionally it is a needless hypertrophy.

Dr E. Olsen: Although we don't know the aetiology there is certainly sufficient clinical differentiation as well as pathological counterparts to make the distinction between the two. From the descriptive point of view these patients are totally different. Now with regard to this argument, from the pathological point of view are congestive cardiomyopathic hearts hypertensive hearts? Dr Oakley has put forward the thesis that they are. If these patients have a brief history and a brief illness, and then go into intractable myocardial failure then, as a pathologist, I think I would be unable to tell the difference. But if hypertension has been present for some time, even for 3-6 months, then some pathological features will be found. Now in none of our patients, whom we have examined in great detail, do we find any changes in the kidney or in the small arteries of the heart themselves. Dr Brockington said that he has not yet analysed all the changes in the small vessels of the coronary arterial tree. If his 
patients had been in heart failure for a long time, one does get some fibrosis purely secondary to the degree of dilatation and it is only in these areas that you get some small vascular changes with internal thickening. As far as can be seen these changes may well be secondary. I, therefore, think that although some patients with congestive cardiomyopathy may fall into the group which Dr Oakley proposes, the majority of patients show no evidence of hypertensive disease either in the heart or elsewhere in the body and I think that the majority have nothing to do with hypertension.

DR C. OAKLEY: Earlier, I defined cardiomyopathy simply as heart muscle disease of unknown cause or association and classified it according to whether the left ventricle is dilated or not, hardly an edifice without foundation. In other words, I hope that in some years' time, we will have eliminated 'cardiomyopathies' because we will only have heart muscle diseases whose origin we know and understand. I classified them further into systolic failure and diastolic failure. Hypertrophy without dilatation, and in the absence of any discernible cause, cannot be due to hypertension because systolic function is often good and the cardiac output normal. They have to go into a separate group and I agree that they do not develop congestive cardiomyopathy. The words congestive cardiomyopathy linger on because we don't know the cause. I am very grateful to my opponent in debate, John Goodwin, for supporting my argument so well! Of course, in his series of congestive cardiomyopathies, there was a low incidence of hypertension because its presence would have earned the appellation 'hypertensive heart disease' and have meant removal from the series of cardiomyopathies! So only a few were allowed to remain on the lists as 'congestive cardiomyopathy' despite mild hypertension. I am grateful to him for pointing out the existence of a spectrum in which admission at one end to the lists of congestive cardiomyopathies depended on the left ventricle having failed sufficiently badly for the causative hypertension to have been lost completely or partly, while maintenance of a more efficient left ventricle enabled hypertension to be recognized.

I would like to make a comment on Dr Dickinson's remarks about noradrenaline infusion and its effect, because his is a very helpful comment. My objection is that the infusion of noradrenaline, a potent increaser of peripheral resistance and also a drug with a beta-action on the heart, initially improves cardiac performance but also increases metabolic demand with an oxygen-wasting effect. Then noradrenaline produces heart failure with a dilated left ventricle, something rather like congestive cardiomyopathy. A very nice piece of evidence. I don't think that Professor Dollery's remarks about denervation have relevance, because we now have a lot of knowledge about the effects of complete denervation of the human heart from the transplantation experience. Shumway's group recently reported on the haemodynamics of the transplanted human heart a year or more after operation and these hearts were proved to remain totally denervated. There was no rate response to amylnitrite, carotid sinus pressure and so on, and these hearts were shown to function remarkably well. They could respond to exercise by an increase in stroke volume and showed a steady increase in minute output in response to exercise, which was on a line parallel with but below that of a normal heart. So I would reject this idea of a humoral origin for congestive cardiomyopathy. I hope that very soon we will be able to remove congestive cardiomyopathy completely into the realms of hypertension.

DR Dickinson: Can we apply pathological evidence?

Dr OAKLEY: I understand that the changes of hypertension are really those of premature ageing, and I always ask my patients-Do you want your arteries to wear out before you do? If so, don't take your tablets. If it is simply a matter of premature ageing I cannot see how 6 months or even 3 or 4 years of premature ageing developing while the blood pressure is high before the left ventricle fails is goinge to be detected even by the most sophisticated pathologists.

DR BARRY (Ipswich): I would like to support Dr Goodwin. The patients seen by Dr Oakley already have some disease or complaint, but many subjects with congestive cardiomyopathy are detected when the patient is not complaining of anything. In survey work over the past 6 or 7 years I have found and followed twenty-three cases of cardiomyopathy. When they eventually died from heart failure, none of the twenty-three had developed hypertension. I have followed quite a few COCM and I would be very surprised if any of those were hypertensive. I would add that I had their kidneys examined.

Dr BROCKINGTON: I would like to join Dr Hollman in the argument against the two people to whom I owe my cardiological training. It would be better if the clinicians left terms like 'heart muscle disease' and 'cardiomyopathy' to the pathologist, who can see the changes in the heart muscle, and that we used words that are strictly clinical or haemodynamic in meaning and have no tendentious associations borrowed from pathology. The term which is often being used in this conference, contractile failure or myocardial failure, is a much better term than congestive cardiomyopathy.

DR Dickinson: The trouble about contractile failure is that nobody can define contractility.

DR Brockington: Well, then it will have to be 
myocardial failure, but we need a term that contains no hidden prejudice in favour of intrinsic myocardial disease and against cardiac overload.

Professor Goodwin: I would like to comment first on Dr Hollman's remarks. I am glad he has raised this question about over-classification, because it certainly is a danger. I recently suggested that we might remove all the things that $I$ have classified as secondary cardiomyopathies and the diseases which have a known origin, leaving us with the hypertrophic obstructive and the congestive. I have really said goodbye to constrictive cardiomyopathy, although I have a feeling that like the phoenix, it may recur from time to time!

Dr Bubanu (Yugoslavia): I would like to describe our experience in congestive cardiomyopathy. We have assembled material on twenty-eight patients in 7 years. One of our patients had post-partum cardiomyopathy after the delivery of twins. In three of our patients, the illness developed after some viral infection. In fourteen of the patients we are convinced that alcohol must have been a very important factor. Eleven of the twenty-eight patients died, a mortality rate of about $39 \%$, and five came to necropsy. The findings were typical of congestive cardiomyopathy. None of these patients were hypertensive during the last 5 or 7 years in which we followed them. They were normotensive, and in the alcoholic group the blood pressure was low all the time. We think that in our country alcohol is a very important factor.

Dr Stiggelbout (Amsterdam): I would like to ask Dr Shaper a question. It is more than a decade since I worked in Africa, and there was then none of this sophisticated blood pressure taking and measuring. I did out-patients with the local policeman controlling the crowds and had to see 400 patients a morning. This gives me the feeling that what is said and written about Africa nowadays, might not be Africa! We might be hearing about highly selected groups of people. I have never seen an African woman 14 days after delivery, because the African woman disappears from the hospital within $48 \mathrm{hr}$. Would you not feel that some of the series that have been reported might be different from what I would call Africa?

DR SHAPER: Patterns have changed over the past decade and are constantly changing. The one thing that we have become very aware of recently is that much of what was reported in the early African literature related strictly to people who came to hospital. We are very careful nowadays to make clear which are strictly hospital studies, as these may not relate to the community in general. In the last decade there have been a number of health centres and district hospitals developed and the universities have established rural health centres where people are followed in their home conditions. I think we now have a better perspective of what is really going on in what you would like to call Africa, i.e. Africa outside the big towns. One should emphasize that even the cities are still Africa! Most of the material that Dr Brockington has described do reflect what is general in the community, and they are not only hospital studies.

DR Brockington: I have been working at University College Hospital, Ibadan and $50 \%$ of the patients attending the hospital come from the urban area of Ibadan, which has just under a million people and the other $50 \%$ come from the surrounding countryside. I don't think that the lives of these patients have been very much changed by modern medicine or other influences. We are probably studying African patients as they have been for a considerable time.

DR Dickinson: In my experience of looking after a lot of hypertensives in the clinic, there are certainly some people who maintain diastolic pressures of $130-140 \mathrm{mmHg}$ for some 10 years (we can't, for various reasons, control them at all well) and who never go into heart failure; their hearts may be relatively small. There are others, and I don't think they are all that rare, who start off severely hypertensive, and over the course of a year, without a definite myocardial infarct, present in the end with a normal blood pressure, some evidence of heart failure and who no longer require drugs for control. This is the sequence which Dr Oakley has described. 